\title{
Contribution De L'apprentissage Automatique Et De La Fouille De Textes À La Construction De Systèmes D'information Pour Exploitants Agricoles
}

\author{
Ines Abdeljaoued-Tej, PhD \\ ESSAI, Université de Carthage, Tunisie, Laboratoire BIMS, LR16IPT09, \\ Institut Pasteur de Tunis, Université Tunis El Manar, Tunisie
}

Doi:10.19044/esj.2020.v16n12p83 ～URL:http://dx.doi.org/10.19044/esj.2020.v16n12p83

\begin{abstract}
Résumé
Les systèmes agricoles vont des techniques intensives aux interventions minimalistes, en passant par le semi-direct. Ces systèmes supposent une connaissance précise des pratiques agricoles par les exploitants et les techniciens. A cela, s'ajoute la maîtrise des nouvelles technologies, le contrôle des resistances aux traitements, l'acquisition des connaissances sur les variétés de semences, l'impact sur les sols, etc. Toute cette masse d'information est disponible sur internet : dans des articles scientifiques, des forums de discussions, des sites web spécialisés et les réseaux sociaux. Ce sont des informations sous format texte, généralement mal structuré. L'objectif de ce travail est de donner une vue générale de la recherche sur la fouille de données textuelles en agriculture. Il présente les principales méthodes permettant l'extraction d'informations pertinentes et teste la fouille sur des données de Scopus, de Twitter et d'un site commercial spécialisé en produits agricoles. Un exemple de classification de données est détaillé, via les algorithmes d'apprentissage automatique. Le code informatique pour réaliser cette revue est sur Python.
\end{abstract}

Keywords: Apprentissage Machine, Extraction D'information, Agriculture Intelligente 


\title{
Contribution of Machine-Learning and Text Mining to the Construction of Farmers' Information Systems
}

\author{
Ines Abdeljaoued-Tej, PhD
}

ESSAI, Université de Carthage, Tunisie, Laboratoire BIMS, LR16IPT09, Institut Pasteur de Tunis, Université Tunis El Manar, Tunisie

\begin{abstract}
There are several techniques used in agricultural systems, from intensive to minimum intervention, no-tillage, and organic methods. Those systems suppose that the farmers have a precise and continuous knowledge of the methods used. Furthermore, the expertise of those new technologies, the control of the treatment resistance, the gain of knowledge on seed varieties and impact on the soil are aspects that should be taken into consideration by farmers who have to keep an eye on the novelties. All those information are available on the internet, in scientific publications, discussion forums, specialized websites, and social media. Resulting from the disorderliness of those text information, the goal of this work is to provide a global view on textual data mining for agriculture. It presents the main methods in extracting relevant information and tests it on data coming from Scopus, Twitter and a website for agricultural products, to illustrate the technique used. Coded in Python, this work provides an example of data classification via machine learning tools.
\end{abstract}

Keywords: Machine Learning, Smart Farming, Text Mining, Web Scraping

\section{Introduction}

L'observation des divers domaines utilisant aussi bien le langage naturel que les ressources techniques les plus modernes, montre que l'agriculture, et plus particulièrement les sciences agronomiques, sont actuellement révolutionnées par l'essor du numérique (Drury and Roche 2019). L'agriculture de précision ou le smart farming permet d'aider à la décision à partir de données locales (Pivoto et al. 2018). Connaitre l'état des plantes et la qualité de la terre, interroger des sources de données distantes (prévision météo, archives, tweets) sont un moyen d'accompagnement des agriculteurs afin de suivre et de prévoir l'évolution des cultures. Dans (Chebrolu et al. 2017) un robot photographie régulièrement des plants de betterave et collecte des informations locales. Ce sont des données qui, 
utilisant des techniques d'apprentissage automatique (principalement des outils statistiques et des réseaux neuronaux), permettent d'aider au suivi du risque de maladies, du développement des cultures, etc. Cette démarche est aussi appliquée pour la commande des automates à distance (irrigation, robots de traite, robots d'alimentation). La décision peut aussi être diffusée vers des systèmes distants comme le lancement d'alertes via des SMS ou des emails (évènement climatique, procédure de traitement lors des infections). Dans (Da Costa et al. 2018) ce sont les différentes maladies du riz et les traitements préconisés qui sont retenus et étudiés. Les effets secondaires et l'évaluation de traitements sont obtenus grâce à un traitement de la langue sur des corpus spécifiques.

L'essor du monde agricole et la modernisation des techniques sont un terreau pour les nouvelles méthodes de fouille textuelle et d'intelligence artificielle, qui trouvent aujourd'hui leur application en agriculture numérique. De plus, l'étude du comportement des techniciens ou des exploitants agricoles permet de reproduire des modèles, grâce à l'intelligence artificielle, où le comportement est une valeur monnayable (Maruşter et al. 2008). Sachant l'importance du traitement des données textuelles, et plus particulièrement, l'extraction de connaissances à partir de textes (blogs, forums de discussion), la fouille de données textuelles apporte déjà des résultats intéressants (Liu et al. 2018). Dans (Comer 2016), c'est l'étude du statut des femmes agricultrices qui est étudié, via une revue des articles de presse. Ce traitement médiatique de la parité, est généralisé à d'autres sources de données et est obtenu de manière systématique, via la fouille automatique. Par exemple, l'analyse des revues littéraires (Zschocke 2019) est désormais chose courante, puisque les données sont à portée de main (voir Scopus par exemple de Elsevier (Burnham 2006)).

Cet article s'intéresse particulièrement aux données textuelles, souvent mal structurées, liées à l'agriculture. Le corpus inhérent à cette thématique est aussi bien technique que pratique. Parmi les études déjà menées, il y a la fouille automatique d'articles scientifiques portant sur l'efficacité des traitements administrés aux plantes (Ricroch et al. 2016). Ce sont les nouveaux challenges dans la recherche en agronomie, comme le montre le nombre croissant d'articles scientifiques sur le sujet. Un autre volet porte sur l'extraction d'informations à partir de données expérimentales du domaine agricole : c'est une approche plus technique puisqu'elle aborde des questions d'ingénierie de la connaissance, le but est de pouvoir les intégrer dans des systèmes d'aide à la décision. Dans (Devyatkin et al. 2018) sont présentés les secteurs les plus touchés par la recherche (une étude sur les résumés d'articles publiés entre 2008 et 2015, où le plus gros des thèmes examinés porte sur la sélection de semences). Tout cela repose sur le génie des connaissances et des 
systèmes $\left(\mathrm{KSE}^{1}\right)$, sur le traitement du langage naturel $\left(\mathrm{NLP}^{2}\right)$ ainsi que sur des approches d'exploration de données guidées par des ressources ontologiques et terminologiques.

Les projets qui visent à fournir de nouveaux outils décisionnels efficaces aident le développement agricole ainsi que la protection de la biodiversité (Basnet and Bang 2018). Plus spécifiquement, il s'agit d'acquérir de nouvelles données, d'extraire des connaissances, d'échanger des données et de communiquer des informations utiles entre différents acteurs impliqués dans les divers domaines de l'agriculture. Les algorithmes de classification et de prévision souvent implémentés sont les machines à vecteurs de support $(\mathrm{SVM})$, les réseaux de neurones $(\mathrm{NN})$, les forêts aléatoires (RF) et l'algorithme Naive Bayes (NB). Une multitude de logiciels de fouille de données existe. Par exemple, Weka (Garner et al. 1995) ou R (R Core Team 2017), qui contient des librairies spécifiques aux données textuelles (Silge and Robinson 2017). A l'image de travaux comme (Bird et al. 2009) pour le traitement automatique de texte, c'est le logiciel Python (Rossum 1995 ; Sanner et al. 1999) qui est retenu pour illustrer les exemples de ce travail.

L'objectif de ce travail est de présenter une revue des outils couramment utilisés dans la fouille textuelle en agriculture. Le reste de ce document est détaillé comme suit : la section 1 montre comment se fait la collecte des données textuelles. La section 1.1 détaille les solutions utilisées, se concentre sur les réseaux sociaux et les sites de commerce électronique (ou e-commerce). La section 1.2 montre la pratique de traitement préliminaire des données. La section 1.3 indique comment sont représentées les données : la conversion des mots en vecteurs, et les vecteurs en matrices par exemple. La section 2 explore l'analyse et la fouille des données textuelles via l'analyse sentimentale. La section 2.1 donne un exemple partant de la collecte à l'analyse des données textuelles, puis la section 2.2 détaille la classification des données textuelles d'un site de e-commerce. Les codes utilisés dans les exemples ont été ajoutés en annexe A (pour le prétraitement) et en annexe B (pour l'annotation, la classification et la prévision).

\section{Quels corpus ?}

Le traitement du langage naturel est un des domaines les plus en vue de l'intelligence artificielle. Il s'agit principalement de la parole et du texte produits, à des quantités faramineuses. Les données textuelles peuvent être sous format de SMS, de tweets, de blogs, de statuts Facebook, de commentaires dans des forum de discussion, de réponses à des questionnaires,

\footnotetext{
${ }^{1}$ Knowledge and Systems Engineering

2 Natural Language Processing
} 
extraits de sites web ou d'articles scientifiques collectés via des API ${ }^{3}$ spécifiques. Il arrive aussi que des corpus différents soient associés pour mieux analyser un texte, un peu à l'image d'un lecteur qui se documente via des livres différents, de plus en plus variés, et qui apprend, grâce à cela, à reconnaître le style des écrivains. Dans notre cas, ça serait les principaux algorithmes manipulés, les diverses techniques relevées, les dernières innovations retenues.

Par exemple, si le choix se fait sur l'étude des traitements de semences, il suffirait de lancer une recherche web en fonction de mots clés adéquats (traitement, fongicides, glyphosate, etc.). Un certain nombre de documents en relation avec ces mots clés sont ainsi obtenus : voir section 1.1 pour un exemple d'extraction de textes. Mais, ce qui est commun aux données textuelles issues du web, c'est qu'elles sont mal structurées : elles peuvent contenir des balises, des méta-data, des liens hypertextes, des mots mal orthographiés, une composition de plusieurs langues, un arrangement de chiffres, de tableaux et de figures, des émoticônes, etc. Dans un premier temps, il est primordial de nettoyer le corpus : enlever les balises, transformer toutes les lettres en minuscule, traduire les mots étrangers dans le texte dans la langue étudiée, corriger les fautes d'orthographes, enlever les ponctuations, éliminer les nombres, etc. A cela, s'ajoute l'atomisation du texte. Ce n'est pas le seul prétraitement fait aux données. Les phrases sont réduites à leur minimum (en enlevant les mots communs). C'est ce qui est démontré en section 1.2. Une fois le corpus de textes défini, le processus d'extraction de l'information consiste en la structuration du texte. C'est la partie la plus importante : le codage du texte. Ceci consiste à représenter le texte sous forme d'un vecteur numérique, voir les détails en section 1.3.

\subsection{Techniques d'extraction (web scraping)}

Construire sa propre base de données passe par des applications et des fonctions spécifiques (voir par exemple les API de Twitter, de Facebook ou de Google pour récupérer des données textuelles). Il est en effet possible de collecter des données textuelles directement sur des sites spécifiques, comme les sites d'e-commerce.

Ainsi est assemblé puis téléchargé un catalogue de produits (machines agricoles, engrais, semences) avec des caractéristiques de chaque élément (des attributs comme la marque, la référence, la catégorie, la description ou toute autre information relative au produit). Avec le choix d'utilisation du logiciel Python, il y a principalement les librairies Scrapy (Myers and McGuffee 2015), BeautifulSoup (Richardson 2007) ou urllib2 (Lawson 2015) qui permettent ce type de traitement. Pareillement, la librairie Selenium

\footnotetext{
${ }^{3}$ API : Application Programming Interface. C'est un ensemble de classes, de fonctions et de méthodes accompagnant un logiciel ou un service web.
} 
(Avasarala 2014) permet une extraction structurée de textes, en spécifiant le site web et le type de navigateur.

Le premier exemple tiré du site Scopus avec la requête suivante a permis de collecter les sources des travaux sur l'agriculture, publiés en libre accès (Open Access ou OA) du 1er janvier au 30 avril 2019: TITLE-ABSKEY(agriculture) AND (LIMIT-TO(PUBYEAR,2019)) AND (LIMITTO(ACCESSTYPE(OA))).

Dans la figure 1 il y a une projection du nombre de publications scientifiques retenues, sur une map-monde des pays d'origine de ces travaux.
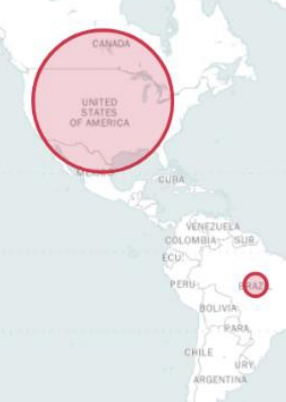

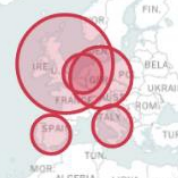

O
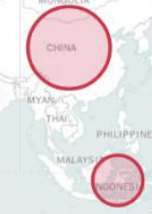

Figure 1 : Publications scientifiques sur l'agriculture, selon le pays ou le territoire. La requête a été limitée au premier trimestre de 2019 et aux journaux en libre accès. Le résultat donne 1892 documents référencés sur Scopus, avec du premier au dernier classement : les Etats Unis avec 360 publications retenues, la Chine, l'Angleterre, l'Indonésie, l'Allemagne, l'Italie, l'Espagne, l'Inde, les Pays-Bas et le Brésil avec 79 publications en libre accès sur l'agriculture

Par ailleurs, il y a moyen de travailler sur le flux de données sur Twitter : ce sont des statuts qui indiquent l'adresse IP (et donc la région ou la localisation géographique) et l'évolution temporelle (selon la saison, le jour de la semaine, l'heure de la journée), suivant l'étude souhaitée sur le corpus. Par exemple, dans (Zipper 2018), partant de tweets collectés dans dix régions des Etats Unis, une estimation des dates de semence pour chaque état a été finalisée, les nouveaux enjeux agricoles (e.g. les conditions météorologiques, les ravageurs des cultures) et les orientations des activités de vulgarisation et de sensibilisation de ces régions ont été tirés pour chaque culture.

Ainsi, la saison ou l'horaire des publications sur le réseau internet a une influence sur l'analyse de l'information collectée. Pour illustrer cela, voir cet exemple de tweets liés à la communauté GMOfree ${ }^{4}$. Les tweets sont extraits durant le premier trimestre de l'année 2019. La base analysée contient

\footnotetext{
${ }^{4}$ Militant contre les organismes génétiquement modifiés
} 
une centaine de tweets. La figure 2 donne l'évolution des appréciations et des partages des divers tweets sur le mois d'avril 2019.

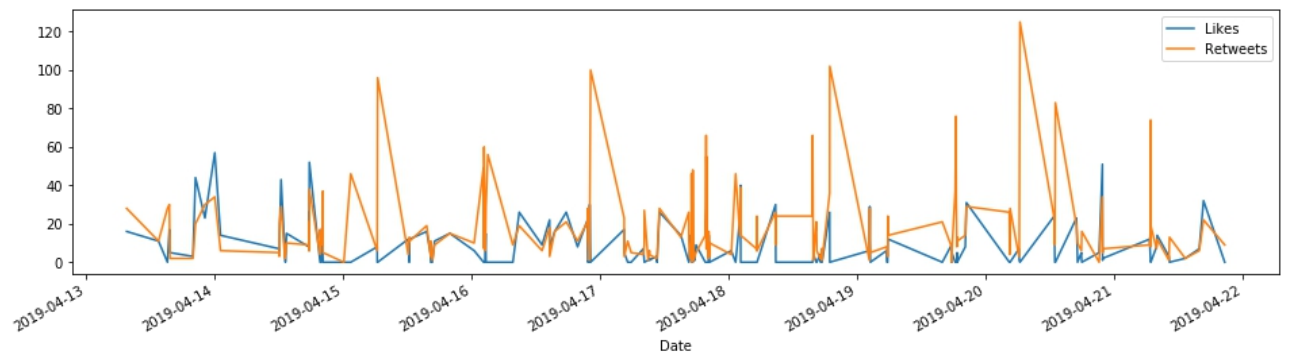

Figure 2 : Comportement des utilisateurs de Twitter sur le mois d'avril 2019 qui s'intéressent à la communauté des militants contre les organismes génétiquement modifiés, connue sous le nom GMOfree. Il y a une corrélation entre le nombre d'appréciations (Likes) et le nombre de partages (Retweets)

Les personnes qui visitent un site sont identifiées via une liste d'intérêts (basée sur les mêmes caractéristiques et enregistrant toute autre information de navigation), et ceci sert par exemple dans la détection des influenceurs (Ghosh et al. 2012). Ce sont aussi des informations utilisées dans la modélisation du comportement des visiteurs, comme c'est le cas dans (Ray 2017) ou dans (Ram et al. 2015) pour l'étude de la régulation de l'eau grâce aux capteurs intelligents en agriculture. D'autres comme (Hunt 2007) utilisent des études classiques, sur le terrain suite à des questionnaires, ou ciblent une recherche spécifique de textes scientifiques sur l'agriculture (Figueroa-Rodríguez et al. 2019).

\subsection{Prétraitement des données}

La nature des données textuelles massives, qui est liée à des objets non structurés (provenant d'une extraction de concepts, des constructions d'ontologies), fait que l'intervention des linguistes via les analyses du langage naturel et de la sémantique, est souvent nécessaire.

Pourtant, l'utilisation des outils de fouille (data mining) qui fonctionnent avec des données structurées, est souvent nécessaire, afin de rendre l'analyse réalisable. Pour les mettre sous une forme adéquate, la transformation des textes est privilégiée (en les rendant mieux hiérarchisés et structurés). Une fois le corpus disponible, il faut procéder à la mise en place d'un dictionnaire (i.e. un ensemble de mots pertinents). Pour cela, les opérations de traitement du langage naturel (NLP) détaillées ci-dessous sont appliquées : la normalisation, l'atomisation, la suppression des mots inutiles, et enfin le retour à la racine des mots. La figure 3 détaille ce processus de prétraitement des données textuelles. 


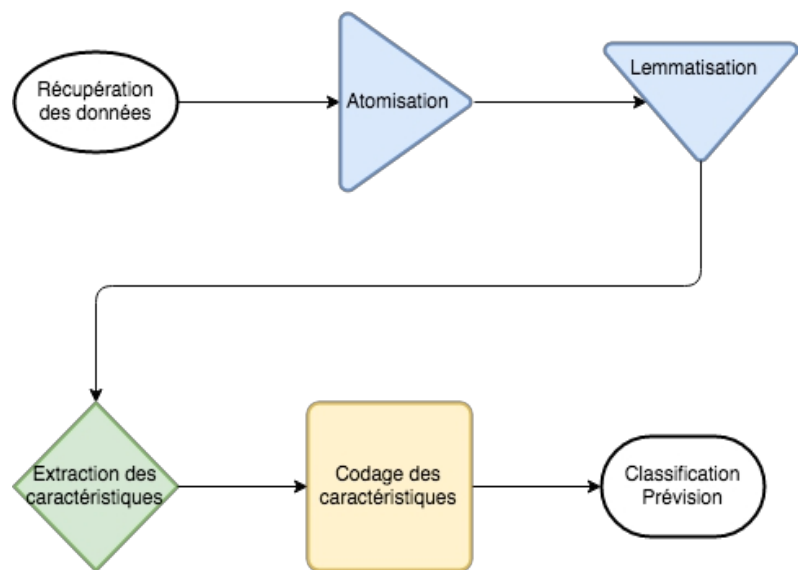

Figure 3 : Méthodologie de fouille des données couramment utilisée dans la littérature. Le texte est transformé en une suite de phrases. C'est la segmentation où s'effectue la recherche de la partie d'un texte qui a le plus de signification par rapport au reste du document. Il s'agit de l'extraction des caractéristiques. A cela, s'ajoute la reconnaissance de mots clés liés à la thématique étudiée. La classification peut se faire en se basant sur les groupes des verbes

\subsubsection{Normalisation du texte}

La normalisation d'un document consiste à rendre l'orthographe le plus uniforme possible. Les opérations à effectuer sont par exemple la conversion en minuscule (Bioud 2006), la transformation des chiffres en mots, la suppression de toute sorte de ponctuation et des espaces de trop entre les mots, la transformation des abréviations en groupes de mots, etc.

\subsubsection{Atomisation}

En anglais, il s'agit de Tokenization i.e. de la conversion d'une phrase en un groupe de mots (verbes, noms, nombres). Après cela, une sélection de mots s'opère. Sont enlevés les mots inutiles, qui n'apportent pas un plus au sens de la phrase (the, $a$, on, de, etc.) ou les mots vides. Ces mots sont appelés stopwords et la librairie NLTK sur Python (Loper and Bird 2002) est souvent utilisée pour les éliminer.

\subsubsection{Racine des mots}

Pour revenir à la racine du mot, il y a plusieurs algorithmes qui éliminent les terminaisons morphologiques communes des mots. Notamment, l'algorithme Porter stemming (Porter 2001) et l'algorithme Lancaster stemming (Hooper and Paice 2005). Il est aussi possible d'utiliser une autre technique se basant sur le sens du mot comme avec NLTK (WordNet Lemmatizer), spaCy, TextBlob, Pattern, gensim, Stanford CoreNLP, MBSP, GATE, Illinois Lemmatizer, et DKPro Core. Voir (Solberg 2012) pour une revue complète de la construction d'un corpus. 
Le système d'indexation des termes produit un résultat pertinent sur l'analyse de textes. Aussi, au dictionnaire de mots, s'ajoute la pondération des termes qui affecte une mesure liée à l'importance d'un mot particulier dans la description d'un produit (par rapport aux descriptions des autres produits). La méthode la plus fréquemment utilisée est la pondération TF-IDF (ou Term Frequency-Inverse Document Frequency) comme expliqué dans (Salton and Buckley 1988). Par exemple, pour la base de tweets collectée dans la section 1.1 , les mots les plus significatifs sont obtenus suite au prétraitement du corpus. Ces mots apparaissant dans le nuage de la figure 4 : cancer, Roundup (qui est la principale marque produisant du glyphosate). La présence du mot Vietnam (qui a interdit le glyphosate en mars 2019) est à souligner.

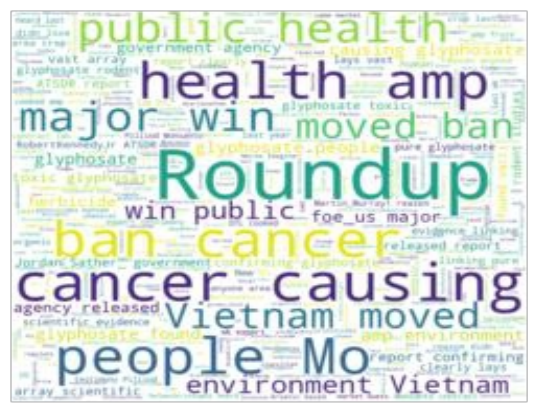

Figure 4 : Nuage de mots généré d'une base de tweets, extraits sur la période allant de 2016 à 2019, suivant les comptes américains, canadiens et européens de @ GMOfree. Seuls les mots dont la fréquence d'apparition dépasse un certain seuil sont conservés

L'idée fondamentale de cette démarche est qu'une personne extérieure au domaine d'étude puisse se faire une idée générale de la base simplement en regardant les nuages de mots.

\subsection{Représentation des données textuelles}

Il est important de regrouper le texte en phrases, les phrases en mots, les mots par thèmes (traitements, machineries, semences, nourritures). Ainsi, chaque texte est représenté par un groupe de mots et ces derniers sont symbolisés par un vecteur (avec leur fréquence d'apparition) dans le dictionnaire lié au corpus. Structurer l'information sous forme de vecteur permet de les fouiller pour relever, par exemple, les mots les plus fréquents. Dans (Agard 2007), le vecteur est construit dans le but de représenter le contenu de la phrase via un vecteur de poids qui se calcule sur l'apparition du terme dans le texte. Ceci est appelé word embedding ou intégration de mots. Le principe de cette procédure est le suivant : chaque coordonné du vecteur représente un mot du texte.

Par exemple, si on considère les deux phrases $\mathrm{P} 1$ et $\mathrm{P} 2$ suivantes : 
P1 : On a ajouté de 1 azote.

P2 : On a une carence en azote.

Alors, le vocabulaire formant ces phrases est :

$\mathrm{V}=\{$ on, a, ajouté, de, 1 , azote, une, carence, en $\}$.

Les deux phrases pourraient être représentées par les vecteurs suivants :

$\mathrm{P} 1=[1,1,1,1,1,1,0,0,0]$.

$\mathrm{P} 2=[1,1,0,0,0,1,1,1,1]$.

Ce modèle simple et efficace est appelé Bag of Words (BoW). Il donne les mots qui sont présents et dans quelle mesure ils sont présents dans des documents codés (sans aucune information sur l'ordre). Il existe de nombreuses façons d'étendre cette méthode simple, à la fois en clarifiant mieux ce qu'est un mot et en définissant ce qu'il faut coder pour chaque mot du vecteur. Ceci permet de décrire la présence de mots dans les données textuelles : par exemple, deux champs de texte similaires contiennent le même type de mots, et auront donc un sac de mots analogues. De plus, le texte seul permet d'apprendre sur la signification du document. BoW est la technique la plus populaire puisqu'à partir d'un vocabulaire, elle permet de choisir une mesure de présence du mot dans ce paquet de mots. Sont obtenues des matrices avec en ligne la phrase, en colonne les mots et les coefficients sont des 0 et des 1 pour présent ou absent dans le dictionnaire des mots.

D'autre part, la fréquence d'un mot $\mathrm{T}$, notée $\mathrm{TF}$, est définie comme le rapport entre le nombre de fois que ce terme $\mathrm{T}$ apparaît dans la phrase et le nombre total de mots dans cette phrase. Un mot $\mathrm{T}$ qui apparaît un grand nombre de fois dans tous les documents, n'est pas d'une grande utilité. Par conséquent, on utilise l'IDF (la fréquence inverse des documents) qui est le logarithme du rapport entre le nombre total de phrases et le nombre de phrases dans lesquelles ce mot $\mathrm{T}$ est présent. En général, pour un terme donné, la quantité TF-IDF est calculée : c'est le produit de TF et de IDF du mot. A cela, s'ajoute le sac de mots $(\mathrm{BoW})$ qui fait référence à la représentation du texte.

Des séquences de $n$ mots adjacents extraites d'une séquence de mots sont également utilisées. Cette combinaison est appelée $n$-gramme et est employée pour la catégorisation des textes (Fürnkranz 1998). Les $n$-grammes avec $n=1$ sont appelés $u n i$-gramme. De même, les bi-grammes $(n=2)$, les trigrammes $(n=3)$ et ainsi de suite peuvent également être utilisés (jusqu'à la phrase complète). Le principe de base des $n$-grammes est qu'ils capturent la structure du langage, comme la lettre ou le mot qui est susceptible de suivre celui donné. Plus $n$ est élevé, plus on donne de l'importance au contexte de la phrase. La longueur optimale dépend de l'application recherchée : c'est un juste équilibre entre les différences importantes dans le texte, et les connaissances générales de ce dernier. Voir par exemple (Socher et al. 2013) pour capturer l'effet de la négation dans une phrase. 
A ces méthodes intuitives et simples, s'ajoutent des méthodes plus sophistiquées et plus variées. Il y a Word2Vec, Glove et FastText où l'incorporation du mot est l'une des représentations les plus populaires du vocabulaire. L'idée est de capturer le contexte d'un mot dans un document, la similarité sémantique et syntaxique, la relation avec d'autres mots, etc. Word2Vec est l'une des techniques les plus populaires d'apprentissage de l'intégration de mots à l'aide de réseaux neuronaux peu profonds. Il a été développé chez Google (Mikolov et al. 2013). La sélection de caractéristiques passe par exemple par les méthodes SkipGram et CBOW (Kou et al. 2015). D'autre part, GloVe (Pennington et al. 2014) est un algorithme d'apprentissage non supervisé permettant d'obtenir des représentations vectorielles de mots. La formation porte sur les statistiques globales de cooccurrence mot-mot issues d'un corpus, et les représentations résultantes présentent d'intéressantes sous-structures linéaires de l'espace vecteur-mot. Enfin, FastText (Mikolov et al. 2018) est une bibliothèque légère, gratuite et à code source ouvert, qui permet aux utilisateurs d'apprendre les représentations et les classificateurs de texte. Cela fonctionne sur du matériel générique standard. Les modèles peuvent ensuite être réduits en taille (Joulin et al. 2016 ; Bojanowski et al. 2017).

Comme mentionné ci-dessus, Word2Vec utilise une architecture de type réseau de neurones. Mais certains codages utilisent des outils de réseaux de neurones profonds comme dans (Bianchini and Scarselli 2014). La recherche dans ce domaine est effectivement très riche : voir (Howard and Ruder 2018) qui présente un parmi les derniers algorithmes utilisés dans la fouille de données textuelles. Voir aussi le codage contextuel dans (Farhan et al. 2016) utilisant la technique de fenêtre dynamique sur les mots. Le plus important dans ce codage est de garder le contexte du mot, pour ne pas perdre la signification dans la phrase.

\section{Analyse sentimentale}

Le métier d'agriculteur est très complexe. Il englobe la gestion quotidienne de l'exploitation (choix des cultures, rendement des parcelles, contraintes en machinerie et en ressources humaines) et une haute technicité pour un suivi de l'évolution des cultures, la détection des maladies à temps, l'utilisation raisonnée des ressources, etc. Les exploitants agricoles doivent être informés sur les variétés de semences qui s'adaptent le mieux à leurs sols. Ils doivent connaître les effets des traitements ainsi que les résistances. 


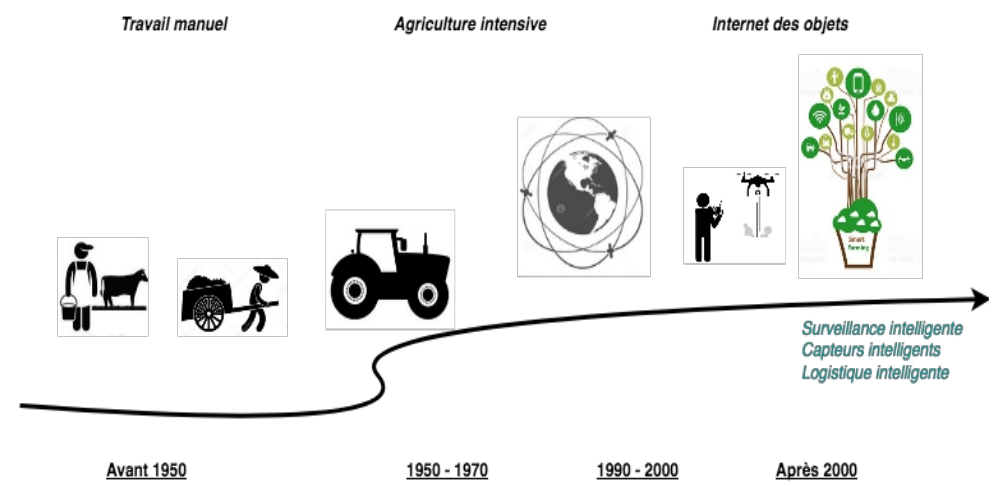

Figure 5 : Évolution des diverses techniques dans le domaine agricole

Les études les plus récentes portent également sur les objets connectés (ou Internet of Things) parce qu'un nombre de plus en plus important de capteurs et d'objets intelligents sont reliés à l'activité agricole. La figure 5 montre l'évolution des techniques agricoles, et l'utilisation des outils de calculs modernes influent sur le rendement de l'agriculture d'aujourd'hui.

C'est dans ce contexte qu'intervient l'analyse sentimentale et plus particulièrement l'analyse de polarité (négative, neutre, positive) et l'analyse d'intensité (faible, moyenne, forte). Sur un corpus donné, ceci permet de construire des alertes via le nombre absolu et relatif de mots négatifs qui paraissent dans un message, un niveau de critique : mot neutre, mot alarmant, mot extrêmement alarmant, une indication de la présence ou de l'absence de ces alertes, etc. Par exemple le degré d'urgence d'un message se calcule en additionnant le nombre de mots critiques, multipliées par leurs niveaux de critique respectifs. Plus le résultat est élevé, plus l'énoncer est alarmant.

Dans la section 2.1 est donné un résultat d'analyse sentimentale du corpus tiré d'un site de e-commerce. L'objectif est de comprendre le comportement des agriculteurs ou des utilisateurs de sites de e-commerce, de leur proposer du contenu adapté à leurs attentes, de prédire leurs besoins futurs (selon les semences achetées, la saison, la localisation géographique de la parcelle exploitée). Il y a des avantages et des inconvénients dans les systèmes basés sur du contenu : suivi des utilisateurs actifs, création d'un profil d'utilisateurs, prise en compte rapidement d'un nouveau produit mis sur le marché. Les inconvénients, pour cette approche basée sur le contenu, sont la nécessité d'une quantité importante d'informations, d'avoir des utilisateurs cloisonnés dans des profils spécifiques, etc.

La section 2.2 présente les algorithmes qui seront utilisés en apprentissage automatique. La section 2.3 donne les performances de ces algorithmes. Il s'agira d'extraire le corpus et d'annoter les descriptions. Puis, est entamé la classification des documents sur la base d'entraînement. Ensuite, la démarche à suivre consiste à effectuer des prévisions pour les nouveaux 
documents, afin de classer correctement les descriptions en trois types (positif, négatif ou bien neutre).

La méthodologie simplifiée est présentée dans la figure 6 ; la base de test étant celle qui permettra de mesurer les performances des divers classificateurs.

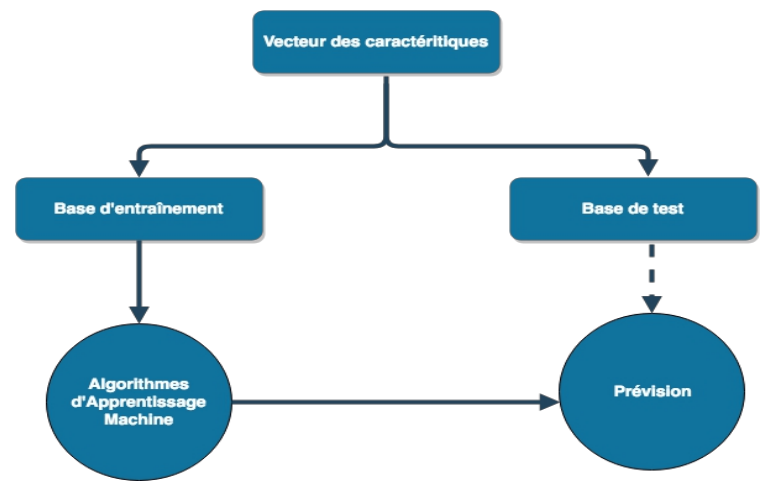

Figure 6 : Modèle simplifié pour l'apprentissage machine

\subsection{Exemple d'un site de e-commerce}

Pour illustrer les techniques présentées dans la section précédente, cet article se base sur un corpus partant du site web agriconomie.com.

Tableau 1 : Aperçu de la base de données récupérée depuis le site web agriconomie.com. Il y a deux colonnes, la première représente le nom du produit (traitement, engrais, semence) et la seconde colonne donne la description du produit

\begin{tabular}{|l|l|}
\hline Nom & Description \\
\hline Urée 46 Granulé - Granulé & $\begin{array}{l}\text { L'Urée 46 est l'engrais azoté idéal pour toutes } \\
\text { les cultures exigeantes en azote. Elle contient } \\
46 \text { unités d'azote uréique soluble dans l'eau, avec } \\
\text { une forme de granulé. L'azote est un élément }\end{array}$ \\
\hline Sulfate d'Ammoniaque .... & $\begin{array}{l}\text { De part ces caractéristiques techniques (diamètre, } \\
\text { densité), son diamètre moyen élevé et sa haute } \\
\text { densité lui permettent d'être épandu en grande } \\
\text { largeur. Observations notables: Pour les céréales : } \\
\text { le nombre de talle est réduit Pour le colza : } \\
\text { On peut observer une déficience en... }\end{array}$ \\
\hline$\vdots$ & \multicolumn{1}{|c}{} \\
\hline
\end{tabular}

Soit un exemple d'une base de 506 produits. Chaque produit est donné par son nom ainsi que sa description comme indiqué dans le tableau 1. Un traitement préliminaire du texte est indispensable : normalisation, atomisation, suppression de stopwords de la langue française et recherche des racines des mots.

Grâce au prétraitement de la base, on peut avoir des informations sur la longueur moyenne des descriptions, les mots les plus fréquents, la présence d'un mot dans les descriptions, etc. Les deux nuages de mots de la colonne nom et de la colonne description sont donnés dans la figure 7. Les mots les 
plus fréquents dans chaque nuage de mots (en dehors de stopwords) illustre les produits vendus sur ce site de e-commerce ainsi que leurs descriptions.

En étudiant automatiquement le corpus, on peut attribuer une classe à chaque description : selon que les mots utilisés soient neutres, positifs ou négatifs. Ainsi trois classes sont obtenues, comme le montre la figure 8. Une description neutre du produit, une description positive et une description négative. En effet, l'utilisation des produits phytosanitaires ou des insecticides nécessite souvent une attention particulière. L'étude des descriptions données dans le corpus permet de détecter de manière systématique les produits dangereux (classés dans "négatif") ou ayant des descriptions neutres ou positives (absence de termes alarmant dans la description du produit).
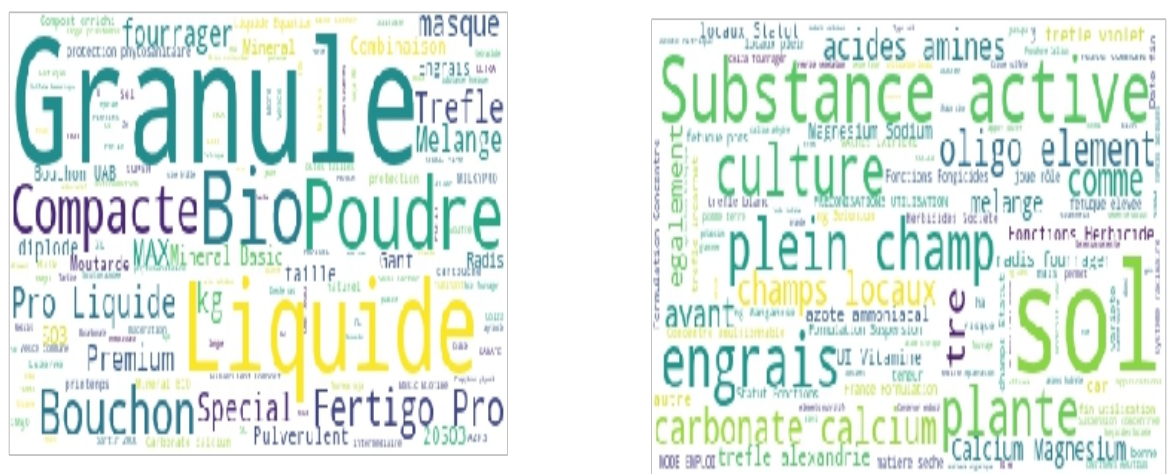

Figure 7 : Nuage de mots représentent les noms des produits ainsi que leurs descriptions. Les mots qui reviennent le plus souvent dans le nom des produits et le type d'engrais sont : liquide, granulé ou en bouchon. Des conseils peuvent être tirés des descriptions comme le stade d'administration du traitement ou les éléments nutritifs constituant les produits

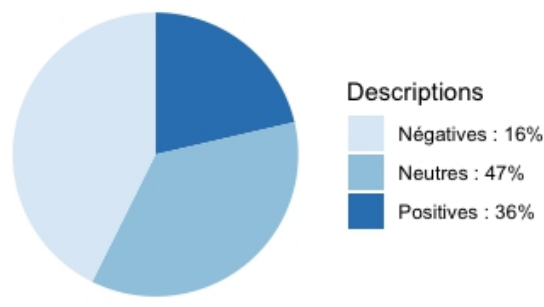

Figure 8: Annotation des descriptions suite à une analyse sentimentale (ou de polarité). Une majorité des descriptions a une tonalité neutre, ce qui est appréciable lorsqu'il s'agit de vente d'insecticides ou d'herbicides. Les descriptions sont à $16 \%$ négatives, ce qui indiquerait une alerte. Le reste des descriptions est positif (36\%)

Après cela, et selon la classification de la description, une alerte pourrait être générée selon la gravité du produit et ses effets sur le sol, la plante ou la personne qui le manipule. Mais pour réussir cela, encore faut-il avoir des documents étiquetés (c'est là où intervient l'analyse sentimentale). 


\subsection{Classification de textes}

L'analyse d'opinion comme présentée dans (Nimirthi et al. 2019) sur des tweets collectés (ou des SMS) passe par une classification des documents. Il y a trois types d'approches : supervisées, non supervisées ou hybrides. En effet, après les mesures à appliquer pour le traitement des données (atomisation, suppression de stopwords et recherche des racines des mots) et une fois que les mots sont plongés dans des vecteurs, les caractéristiques sont extraites. Elles représentent l'entrée des outils de classification des genres (Balikas et al. 2017).

Ici sont présentés quatre exemples d'algorithmes d'apprentissage : SVM, les réseaux de neurones artificiels, les forêts aléatoires et l'algorithme Naive Bayes. Ce sont des méthodes largement utilisées en apprentissage automatique (Patel and Patel 2014; Ramesh et al. 2011) et qui s'adaptent bien au cas de la fouille textuelle.

\subsubsection{Machines à vecteurs de support (SVM)}

Les machines à vecteurs de support (ou SVM) sont des classificateurs linéaires généralisés. Ils minimisent simultanément l'erreur de classification et maximisent la marge géométrique (appelés classificateurs de marge maximale). L'algorithme SVM a été introduit pour la première fois par (Vapnik 1963), puis étendu pour inclure l'astuce du noyau (Vapnik 1999). La motivation d'un SVM est de trouver un hyperplan dans un espace de grande dimension pouvant être utilisé pour la classification. Le but est de trouver une bonne séparation par l'hyperplan en gardant la plus grande distance (marge fonctionnelle). L'intuition est qu'une marge fonctionnelle plus grande entraîne une erreur de généralisation plus faible pour le classificateur. Si $x$ est le vecteur de données et $y$ son label, $w$ le vecteur normal à l'hyperplan $w \cdot x-b=0$, alors il s'agira de résoudre le problème d'optimisation (vu en terme de lagrangien) : $\arg \min _{w, b} \max _{\alpha \geq 0}\left\{1 / 2\|w\|^{2}-S_{i} \alpha_{i}\left(y\left(w \cdot x_{i}-b\right)\right)\right\}$.

\subsubsection{Réseaux de neurones (NN)}

Les réseaux de neurones sont utilisés dans les diverses étapes de fouille de textes. Un réseau de neurones contient au moins deux couches : une couche d'entrée $x$ et une couche de sortie $y$. Il peut contenir des couches intermédiaires de neurones qu'on appelle couches cachées. Les neurones dans les diverses couches sont reliés par des arcs. Chaque arc a un poids appelé poids synaptique.

L'objectif de l'algorithme est de chercher les poids synaptiques optimaux qui assurent que la sortie du réseau de neurones $y=f\left(S w_{i, j} x_{j}\right)$ corresponde à ce qui est observé.

La fonction $f$ est appelée fonction d'activation. Il y a une multitude de fonctions d'activation comme la fonction sigmoïde ou la fonction tanh. Dans 
les simulations de cet article, est choisie la fonction ReLu définie par $f(z)=0$ si $z<0$ et $f(z)=z$ sinon.

Par exemple, le modèle prédicatif pour l'apprentissage des word embeddings à partir de texte brut est donné par Word2 $\mathrm{Vec}$ et il utilise un réseau de neurones à deux couches cachées. Plus généralement, il y a moyen d'utiliser des réseaux de neurones graphiques dans la fouille de textes, surtout lorsque les données sont volumineuses (Scarselli et al. 2009). Mais, les réseaux de neurones sont des boîtes noires qui donnent des modèles avec un risque de sur-apprentissage.

\subsubsection{Forêts aléatoires $(\mathrm{RF})$}

L'algorithme d'apprentissage via les forêts aléatoires (connu sous l'abréviation RF pour Random Forest) est une combinaison de plus d'un classificateur, avec de nombreux avantages tels que l'efficacité, plus de variables d'entrées manipulées, l'importance des variables, la robustesse au bruit ainsi que les valeurs aberrantes (Liaw et al. 2002). Il aide à classer les variables en régression ou en classification. Dans (Lebourgeois et al. 2017) il est utilisé pour la classification de données satellites des cartes d'utilisation des terres d'une zone agricole de petits exploitants à Madagascar. Dans (Achat et al. 2016) c'est une compilation de la littérature qui est utilisée dans la compréhension du cycle du Phosphore dans les sols de forêts françaises.

\subsubsection{Algorithme Naive Bayes (NB)}

Cet algorithme se base sur le théorème de Bayes qui est fondamentalement lié aux probabilités conditionnelles. Si A et B sont deux évènement, alors la probabilité que l'évènement $A$ se réalise sachant que l'évènement $\mathrm{B}$ s'est déjà réalisé est donnée par : $\mathrm{P}(\mathrm{A} \mid \mathrm{B})=(\mathrm{P}(\mathrm{B} \mid \mathrm{A}) \mathrm{P}(\mathrm{A}))$ / $\mathrm{P}(\mathrm{B})$.

Lorsqu'il y a plusieurs variables (comme c'est le cas dans un texte), l'approche de l'algorithme Naive Bayes consiste à prendre en considération ces variables indépendamment les unes des autres. L'algorithme s'appuie sur la fréquence des occurrences des mots dans le texte pour en définir la catégorie (Ting et al. 2011). Lors de la classification, l'algorithme suppose que les mots du texte apparaissent indépendamment les uns des autres. C'est une hypothèse forte et le terme Naive vient de cette supposition.

\subsection{Mesures d'évaluation}

Il y a un ensemble de mesures d'évaluation standard largement utilisées dans l'analyse des données. Ces mesures servent à quantifier les performances d'un algorithme de classification binaire supervisé, quelle que soit la tâche. Les mêmes mesures peuvent toujours être utilisées pour la classification multi-classe en divisant l'évaluation en $\mathrm{C}$ classifications 
binaires, où $\mathrm{C}$ est l'ensemble des classes. Ici $\mathrm{C}=3$ pour la description négative, neutre ou positive. La métrique de performance la plus élémentaire est l'exactitude (ou Accuracy), qui mesure simplement la proportion d'instances prédites correctement par le classificateur. Si TP est le nombre de vrais positifs, FP celui des faux positifs, TN celui des vrais négatifs et FN celui des faux négatifs, alors Accuracy $=(\mathrm{TP}+\mathrm{TN}) /(\mathrm{TP}+\mathrm{FP}+\mathrm{TN}+\mathrm{FN})$.

La précision $\mathrm{P}$ mesure la proportion d'instances classées positivement correctement prédites par le classificateur, tandis que le rappel $\mathrm{R}$ mesure la proportion d'instances positives dans les données que le classificateur peut prédire. Ils sont définis comme suit : $\mathrm{P}=\mathrm{TP} /(\mathrm{TP}+\mathrm{FP})$ et $\mathrm{R}=\mathrm{TP} /(\mathrm{TP}+\mathrm{FN})$.

Il est souvent pratique de combiner $\mathrm{P}$ et $\mathrm{R}$ en utilisant la moyenne harmonique ; cette mesure est connue sous le nom de $\mathrm{F}_{1}$-score $=2 \mathrm{PR} /(\mathrm{P}+\mathrm{R})$.

L'évaluation de la méthodologie de classification se fait grâce aux mesures de performance standard (précision, rappel, exactitude et $\mathrm{F}_{1}$-score). Appliquée à la base de données récupérée depuis le site agriconomie.com (labélisée grâce à l'analyse sentimentale), on compare les quatre algorithmes d'apprentissage automatique : SVM, NB, NN et RF. Les performances obtenues sont détaillées dans le tableau 2, qui indique l'exactitude, la précision, le rappel et le $\mathrm{F}_{1}$-score.

Tableau 2 : Performance des algorithmes de classification. On a testé plusieurs architectures pour le perceptron multicouches NN. Le plus performant est le réseau de neurones avec 2 couches cachées, contenant 5 neurones pour la première couche et 10 neurones pour la couche suivante

\begin{tabular}{|c|c|c|c|c|}
\hline Algorithme & Accuracy & $\mathrm{P}$ & $\mathrm{R}$ & $\mathrm{F}_{1}$-score \\
\hline RF & 0.85 & 0.87 & 0.85 & 0.86 \\
\hline SVM & 0.85 & 0.86 & 0.85 & 0.85 \\
\hline NB & 0.78 & 0.82 & 0.78 & 0.79 \\
\hline NN & $\mathbf{0 . 8 7}$ & 0.87 & 0.87 & 0.87 \\
\hline
\end{tabular}

Sur cet échantillon, c'est l'algorithme basé sur les réseaux de neurones qui est le plus performant, comparé aux autres, et compte-tenu des données utilisées. Ces résultats pourraient être améliorés sur une base de données plus grande. Les performances de l'algorithme Naive Bayes pourraient être perfectionnées en suivant le procédé détaillé dans (Kim et al. 2006).

De plus, il est important de relever qu'à ce stade, le principal problème dans l'annotation automatique de texte, utilisant l'analyse sentimentale, c'est l'ambiguïté de certaines phrases ou de certains mots (retrouvée dans la classe neutre). Si la manière d'annoter le corpus était plus fine, la classe neutre serait sans doute réduite au profit des classes positif et négatif. L'optimisation de cette partie est illustrée par exemple dans (Weichselbraun et al. 2013).

Outre l'apprentissage machine, il y a des architectures plus élaborées de types réseaux de neurones profonds. Ce sont des techniques développées pour l'insertion contextuelle utilisée dans la prédiction (Li et al. 2017). Il y a aussi 
(Gardner et al. 2018) qui utilise de l'apprentissage profond, avec des architectures se basant sur des réseaux de neurones comme le bi-LSTM (Baziotis et al. 2017). Une librairie disponible sur git-hub est dans (Pressel et al. 2018).

\section{Conclusion}

Le monde agricole est confronté à des problèmes tels que le manque de successeurs et l'augmentation du nombre d'agriculteurs âgés. Dans (Shimoguchi et al. 2015), c'est une solution qui est apportée pour attirer et offrir des possibilités d'apprentissage aux jeunes générations pour qu'elles deviennent de nouveaux agriculteurs. Ce sont des modèles d'optimisation de la gestion agricole qui sont recherchés. Elaborer puis appuyer la prise de décisions concernant les activités agricoles, notamment la plantation, la récolte, la manutention, le séchage et l'entreposage devient indispensable, surtout que les nouveaux exploitants ne sont pas forcément familiers avec des techniques agricoles basées sur l'expérience (Shikuku et al. 2019).

Dans (Liao et al. 2015), l'exploration de texte est utilisée pour identifier les calendriers de plantation et de récolte pour différentes cultures dans différentes régions des USA. Ainsi, les récoltes peuvent être optimisées lorsqu'on met en place un moyen d'extraction de l'information en temps quasi réel afin de déterminer le moment de l'ensemencement des cultures et les calendriers de récoltes à partir de bases de données accessibles au public.

Explorer autrement les articles de recherches, en utilisant des systèmes $d$ 'informations performant mais simple à utiliser est aujourd'hui possible : par exemple, pour déterminer la dose optimale d'un engrais, la fouille de l'information peut se faire à deux niveaux, celle à l'échelle des utilisateurs et celle des scientifiques (Diallo et al. 2016). Combiner les deux est aujourd'hui possible, avec la généralisation de l'utilisation des réseaux sociaux. D'autres utilisation sont possible, comme l'intérêt d'une étude sur les biocarburants (Danebe et al. 2019) peut être généralisée par une fouille textuelle automatique afin d'étudier les dernières avancées sur le sujet (fouille automatique des résumés d'articles sur le sujet). La qualité de remèdes à base de plantes peut tenir compte des interactions sur les réseaux sociaux et seraient combinées à des études plus classiques en Phytothérapie comme par exemple (Amoussou et al. 2019). Plusieurs autres études pourraient faire partie d'un ensemble plus large, pour la mise en place d'un système d'information destiné aux exploitants agricoles : dans (Arroud et al. 2020) est démontré l'efficacité sur les individus de telles mesures.

Les travaux présentés détaillent l'intérêt et l'apport de l'intelligence artificielle et la fouille textuelle au domaine de l'agriculture numérique ou dans les sciences agronomiques. Les alertes des maladies et le calendrier des traitements peuvent être obtenus systématiquement grâce à une fouille de 
bases de données judicieuses. D’autre part, le travail préliminaire mené sur la fouille de sites web pourrait être utilisé afin de développer un moteur d'avis et de conseils pour une plate-forme de vente agricole. Des recommandations basées sur le contenu, sur la collaboration (notes des utilisateurs) et hybrides (Sundermann et al. 2019) pourraient être intégré à cette solution.

\section{Remerciements}

Nos vifs remerciements sont adressés à Asma Bouhafs pour ses remarques judicieuses et Naceur Tej pour son regard avisé de professionnel en agriculture, curieux des nouvelles technologies. Nos remerciements vont à l'évaluateur anonyme. Aussi, ce travail s'est fait en marge des encadrements de Sayf Chagtmi, Samar Hannachi et Ahmed Saad, sur la fouille de textes, réalisés au sein de l'école supérieure de la statistique et de l'analyse de l'information de l'Université de Carthage en Tunisie.

\section{References :}

1. Achat, D. L., Pousse, N., Nicolas, M., Brédoire, F., and Augusto, L. (2016). Soil properties controlling inorganic phosphorus availability : general results from a national forest network and a global compilation of the literature. Biogeochemistry, 127(2-3) :255-272.

2. Agard, B. (2007). Extraction de connaissances à partir de données textuelles - vue d'ensemble. In 10ème Colloque National AIP PRIMECA, La Plagne, pages 1-10.

3. Amoussou, B. F., Badoussi, M. E., Houndji, S. (2019). Essai de Production d'extrait Bioactif et Bioaromatisant à Partir des Racines de Mondia whitei (Hook f.) Skeels (Apocynaceae) d'écologie Béninoise, European Scientific Journal (ESJ), Volume 15 No 21. Doi:10.19044/esj.2019.v15n21p339

4. Arroud, M., Elkharraz, A., Boubker, O. (2020). Contribution des Systèmes d'Information à la Performance Individuelle des Logisticiens. Le Rôle de l'Implication et de la Justice Organisationnelle, European Scientific Journal (ESJ), Volume 16 No 1. Doi:10.19044/esj.2020.v16n1p138

5. Avasarala, S. (2014). Selenium WebDriver practical guide. Packt Publishing Ltd.

6. Balikas, G., Moura, S., and Amini, M.-R. (2017). Multitask learning for fine-grained twitter sentiment analysis. In Proceedings of the 40th international ACM SIGIR conference on research and development in information retrieval, pages 1005-1008. ACM.

7. Basnet, B. and Bang, J. (2018). The state-of-the-art of knowledgeintensive agriculture : A review on applied sensing systems and data analytics. Journal of Sensors. 
8. Baziotis, C., Pelekis, N., and Doulkeridis, C. (2017). Datastories at semeval-2017 task 4 : Deep lstm with attention for message-level and topic-based sentiment analysis. In Proceedings of the 11th International Workshop on Semantic Evaluation (SemEval-2017), pages 747-754.

9. Bianchini, M. and Scarselli, F. (2014). On the complexity of neural network classifiers : A comparison between shallow and deep architectures. IEEE transactions on neural networks and learning systems, 25(8) :1553-1565.

10. Bioud, M. (2006). Une normalisation de l'emploi de la majuscule et sa représentation formelle pour un système de vérification automatique des majuscules dans un texte. $\mathrm{PhD}$ thesis, Sciences du langage Besançon. Thèse de doctorat dirigée par Cardey-Greenfield, Sylviane.

11. Bird, S., Klein, E., and Loper, E. (2009). Natural language processing with Python : analyzing text with the natural language toolkit. O’Reilly Media, Inc.".

12. Bojanowski, P., Grave, E., Joulin, A., and Mikolov, T. (2017). Enriching word vectors with subword information. Transactions of the Association for Computational Linguistics, 5 :135- 146.

13. Burnham, J. F. (2006). Scopus database : a review. Biomedical digital libraries, 3(1) :1.

14. Chebrolu, N., Lottes, P., Schaefer, A., Winterhalter, W., Burgard, W., and Stachniss, C. (2017). Agricultural robot dataset for plant classification, localization and mapping on sugar beet fields. The International Journal of Robotics Research.

15. Comer, C. (2016). Don't ruffle feathers. timid changes $f$ in the representation of women in the agricultural press in brittany [ne pas faire mauvais genre .les timides mutations de la représentation des femmes dans la presse agricole bretonne]. Etudes Rurales, 198(2) :7795.

16. Da Costa, E., Tjandrasa, H., and Djanali, S. (2018). Text mining for pest and disease identi- fication on rice farming with interactive text messaging. International Journal of Electrical and Computer Engineering, 8(3) :1671-1683.

17. Danebe, K. A., Djeumako B., Beda T., Samon J.B. (2019). Propriétés Physiques et Mécaniques des Graines et Amandes de Jatropha curcas L., European Scientific Journal (ESJ), Volume 15, No 36. Doi:10.19044/esj.2019.v15n36p81

18. Devyatkin, D., Nechaeva, E., Suvorov, R., and Tikhomirov, I. (2018). Mapping the research landscape of agricultural sciences. Foresight and STI Governance, 12(1) :69-78. 
19. Diallo, M.D., Toure, A., Mbacke, F.D., Saleh M.M., Touroumgaye, G., Ndiaye, A.B., Ndiaye, N.D., Diop, A., Guisse, A. (2016). Détermination De La Dose Optimale D'engrais Minéral 1515-15 Sur Cinq (05) Variétés De Maïs Doux (Zea Mays L. ssp. saccharata) Au Sénégal, European Scientific Journal (ESJ), Volume 12, No 27. Doi: 10.19044/esj.2016.v12n27p135

20. Drury, B. and Roche, M. (2019). A survey of the applications of text mining for agriculture. Computers and Electronics in Agriculture, 163 :104864.

21. Farhan, W., Wang, Z., Huang, Y., Wang, S., Wang, F., and Jiang, X. (2016). A predictive model for medical events based on contextual embedding of temporal sequences. JMIR medical informatics, 4(4).

22. Figueroa-Rodríguez, K. A., Álvarez-Ávila, M. d. C., Hernández Castillo, F., Schwentesius Rin- dermann, R., and Figueroa-Sandoval, B. (2019). Farmers' market actors, dynamics, and attributes : A bibliometric study. Sustainability, 11(3):745.

23. Fürnkranz, J. (1998). A study using n-gram features for text categorization. Austrian Research Institute for Artifical Intelligence, $3(1998): 1-10$.

24. Gardner, M., Grus, J., Neumann, M., Tafjord, O., Dasigi, P., Liu, N., Peters, M., Schmitz, M., and Zettlemoyer, L. (2018). Allennlp : A deep semantic natural language processing platform. arXiv preprint arXiv $: 1803.07640$.

25. Garner, S. R. et al. (1995). Weka : The waikato environment for knowledge analysis. In Proceedings of the New Zealand computer science research students conference, pages 57-64.

26. Géron, A. (2019). Hands-on machine learning with Scikit-Learn and TensorFlow : concepts, tools, and techniques to build intelligent systems. " O’Reilly Media, Inc.".

27. Ghosh, S., Viswanath, B., Kooti, F., Sharma, N. K., Korlam, G., Benevenuto, F., Ganguly, N., and Gummadi, K. P. (2012). Understanding and combating link farming in the twitter social network. In Proceedings of the 21 st international conference on World Wide Web, pages 61-70. ACM.

28. Hooper, R. and Paice, C. (2005). The lancaster stemming algorithm. University of Lancester.

29. Howard, J. and Ruder, S. (2018). Universal language model finetuning for text classification.

30. Hunt, A. R. (2007). Consumer interactions and influences on farmers' market vendors. Renewable agriculture and food systems, 22(1) :5466. 
31. Joulin, A., Grave, E., Bojanowski, P., and Mikolov, T. (2016). Bag of tricks for efficient text classification. arXiv preprint arXiv $: 1607.01759$.

32. Kim, S.-B., Han, K.-S., Rim, H.-C., and Myaeng, S. H. (2006). Some effective techniques for naive bayes text classification. IEEE Transactions on Knowledge and Data Engineering, 18(11) :14571466.

33. Kou, W., Li, F., and Baldwin, T. (2015). Automatic labelling of topic models using word vectors and letter trigram vectors. In Zuccon, G., Geva, S., Joho, H., Scholer, F., Sun, A., and Zhang, P., editors, Information Retrieval Technology, pages 253-264, Cham. Springer International Publishing.

34. Lawson, R. (2015). Web scraping with Python. Packt Publishing Ltd.

35. Lebourgeois, V., Dupuy, S., Vintrou, É., Ameline, M., Butler, S., and Bégué, A. (2017). A combined random forest and obia classification scheme for mapping smallholder agriculture at different nomenclature levels using multisource data (simulated sentinel-2 time series, vhrs and dem). Remote Sensing, 9(3):259.

36. Li, L., Jing, H., Tong, H., Yang, J., He, Q., and Chen, B.-C. (2017). Nemo : Next career move prediction with contextual embedding. In Proceedings of the 26th International Conference on World Wide Web Companion, WWW '17 Companion, pages 505-513, Republic and Canton of Geneva, Switzerland. International World Wide Web Conferences Steering Committee.

37. Liao, W.-T., Rodriguez, L., Diesner, J., and Lin, T. (2015). Improving farm management optimization : Application of text data analysis and semantic networks. In American Society of Agricultural and Biological Engineers Annual International Meeting 2015, volume 4, pages 3040-3049.

38. Liaw, A., Wiener, M., et al. (2002). Classification and regression by randomforest. R news, 2(3) :18-22.

39. Liu, Z., Chen, Y., Dai, Y., Guo, C., Zhang, Z., and Chen, X. (2018). Syntactic and semantic features based relation extraction in agriculture domain. Lecture Notes in Computer Science (including subseries Lecture Notes in Artificial Intelligence and Lecture Notes in Bioinformatics), 11242 LNCS :252-258.

40. Loper, E. and Bird, S. (2002). Nltk : the natural language toolkit. arXiv preprint cs/0205028.

41. Mikolov, T., Grave, E., Bojanowski, P., Puhrsch, C., and Joulin, A. (2018). Advances in pre-training distributed word representations. In Proceedings of the Eleventh International Conference on Language Resources and Evaluation (LREC-2018), pages 1-4. 
42. Mikolov, T., Sutskever, I., Chen, K., Corrado, G. S., and Dean, J. (2013). Distributed represen- tations of words and phrases and their compositionality. In Advances in neural information processing systems, pages 3111-3119.

43. Myers, D. and McGuffee, J. W. (2015). Choosing scrapy. Journal of Computing Sciences in Colleges, 31(1):83-89.

44. Maruşter, L., Faber, N., Jorna, R., and Van Haren, R. (2008). A process mining approach to analyse user behaviour. In WEBIST 2008 - 4th International Conference on Web Information Systems and Technologies, Proceedings, volume 2, pages 208-214.

45. Nimirthi, P., Venkata Krishna, P., Obaidat, M., and Saritha, V. (2019). A framework for sentiment analysis based recommender system for agriculture using deep learning approach. SpringerBriefs in Applied Sciences and Technology, pages 59-66.

46. Patel, H. and Patel, D. (2014). A brief survey of data mining techniques applied to agricultural data. International Journal of Computer Applications, 95(9).

47. Pennington, J., Socher, R., and Manning, C. (2014). Glove : Global vectors for word representation. In Proceedings of the 2014 conference on empirical methods in natural language processing (EMNLP), pages 1532-1543.

48. Pivoto, D., Waquil, P. D., Talamini, E., Finocchio, C. P. S., Corte, V. F. D., and de Var- gas Mores, G. (2018). Scientific development of smart farming technologies and their appli- cation in brazil. Information Processing in Agriculture, 5(1):21 - 32.

49. Porter, M. F. (2001). Snowball : A language for stemming algorithms.

50. Pressel, D. M., Choudhury, S. R., Lester, B., Zhao, Y., and Barta, M. (2018). Baseline : A library for rapid modeling, experimentation and development of deep learning algorithms targeting nlp. In Association for Computational Lingustics, pages 1-7.

51. R Core Team (2017). R : A Language and Environment for Statistical Computing. R Foundation for Statistical Computing, Vienna, Austria.

52. Ram, V. V. H., Vishal, H., Dhanalakshmi, S., and Vidya, P. M. (2015). Regulation of water in agriculture field using internet of things. In 2015 IEEE Technological Innovation in ICT for Agriculture and Rural Development (TIAR), pages 112-115.

53. Ramesh, V., Ramar, K., et al. (2011). Classification of agricultural land soils : a data mining approach. Agricultural Journal, 6(3) :82-86.

54. Ray, P. P. (2017). Internet of things for smart agriculture : Technologies, practices and future direction. Journal of Ambient Intelligence and Smart Environments, 9(4):395-420.

55. Richardson, L. (2007). Beautiful soup documentation. 
56. Ricroch, A., Harwood, W., Svobodová, Z., Sági, L., Hundleby, P., Badea, E., Rosca, I., Cruz, G., Salema Fevereiro, M., Marfà Riera, V., Jansson, S., Morandini, P., Bojinov, B., Cetiner, S., Custers, R., Schrader, U., Jacobsen, H.-J., Martin-Laffon, J., Boisron, A., and Kuntz, M. (2016). Challenges facing european agriculture and possible biotechnological solutions. Critical Reviews in Biotechnology, 36(5) :875-883.

57. Rossum, G. (1995). Python reference manual. Technical report, Amsterdam, The Netherlands, The Netherlands.

58. Salton, G. and Buckley, C. (1988). Term-weighting approaches in automatic text retrieval. Information Processing and Management, 24(5):513 - 523 .

59. Sanner, M. F. et al. (1999). Python : a programming language for software integration and development. J Mol Graph Model, 17(1) :5761.

60. Scarselli, F., Gori, M., Tsoi, A. C., Hagenbuchner, M., and Monfardini, G. (2009). The graph neural network model. IEEE Transactions on Neural Networks, 20(1):61-80.

61. Shikuku, K. M., Pieters, J., Bulte, E., and Läderach, P. (2019). Incentives and the Diffusion of Agricultural Knowledge : Experimental Evidence from Northern Uganda. American Journal of Agricultural Economics, 101(4) :1164-1180.

62. Shimoguchi, N., Inaizumi, H., Yasue, H., and Omuro, K. (2015). Impact of farm-based learning practices on young farmers : Case from an organic farm in ogawa town, saitama prefec- ture, japan. Journal of the International Society for Southeast Asian Agricultural Sciences, 21(2) :143-167.

63. Silge, J. and Robinson, D. (2017). Text mining with R : A tidy approach. " O’Reilly Media Inc.".

64. Socher, R., Perelygin, A., Wu, J., Chuang, J., Manning, C. D., Ng, A., and Potts, C. (2013). Recursive deep models for semantic compositionality over a sentiment treebank. In Pro- ceedings of the 2013 conference on empirical methods in natural language processing, pages $1631-1642$.

65. Solberg, L. J. (2012). A corpus builder for wikipedia. Master's thesis, Department of Informatics, University of Oslo.

66. Sundermann, C., Domingues, M., Sinoara, R., Marcacini, R., and Rezende, S. (2019). Using opinion mining in context-aware recommender systems : A systematic review. Information (Switzerland), 10(2). 
67. Ting, S., Ip, W., and Tsang, A. H. (2011). Is naive bayes a good classifier for document classification. International Journal of Software Engineering and Its Applications, 5(3) :37- 46.

68. Vapnik, V. (1963). Pattern recognition using generalized portrait method. Automation and remote control, 24 :774-780.

69. Vapnik, V. N. (1999). An overview of statistical learning theory. IEEE transactions on neural networks, 10(5):988-999.

70. Weichselbraun, A., Gindl, S., and Scharl, A. (2013). Extracting and grounding contextualized sentiment lexicons. IEEE Intelligent Systems, 28(2) :39-46.

71. Zipper, S. C. (2018). Agricultural research using social media data. Agronomy Journal, 110(1) :349-358.

72. Zschocke, T. (2019). Enriching metadata in an international agroforestry research repository with funding-related information by mining grey literature. In Procedia Computer Science, volume 146, pages $112-122$.

\section{Annexes}

\section{A. Prétraitement des données}

Afin de produire sa propre base de données selon un thème d'études choisi (traitement, semence, qualité du sol, irrigation, etc.), la première partie consiste à récupérer les données du web.

\section{a. Extraction des données}

Les librairies utilisées pour charger les données textuelles du web sont importées grâce au code ci-dessous :

from selenium import webdriver

import pandas as pd

from selenium.common.exceptions import NoSuchElementException import re

Les données sont récupérées depuis le site web agriconomie.com et notre navigateur web est Chrome. Le principe est de télécharger les données textes des divers pages web, de manière structurée. Les données sont enregistrées dans data qui a deux colonnes : nom et description.

lien $\quad$ = ['https://www.agriconomie.com/engrais/', 'https://www.agriconomie.com/semences/', 'https://www.agriconomie.com/phyto',

'https://www.agriconomie.com/nutrition', 'https://www.agriconomie.com/bio']

En vue de manipuler les données, c'est la librairie pandas qui est utilisée. La base de données comprend deux colonnes : une colonne nom présentant le produit (engrais, semences, etc.) et une colonne description décrivant le produit agricole. 
import pandas as pd

data $=$ pd.DataFrame.from_records(data,columns $=$ ['nom','description'] $)$

\section{b. Prétraitement de la base}

La préparation des données commence par la normalisation du texte. Pour enlever les accents, utiliser par exemple le code suivant :

import numpy as np

from text_unidecode import unidecode

import unicodedata

def enlever_accents(text):

try:

$$
\text { text }=\text { unicode(text, 'utf-8') }
$$

except (TypeError, NameError):

text = unicodedata.normalize('NFD', text)

text $=$ text.encode('ascii', 'ignore')

text $=$ text.decode ("utf-8")

return $\operatorname{str}(\mathrm{text})$

Ensuite, enlever les mots qui n'ont pas d'impact sur le sens des phrases pour la langue française (stopwords) ainsi que les ponctuations.

from nltk.corpus import stopwords

stop = stopwords.words('french')

data['stopwords'] = data['nom'] $\operatorname{apply}($ lambda $\mathrm{x}$ : len([x for $\mathrm{x}$ in $\mathrm{x} \cdot \operatorname{split}()$ if $\mathrm{x}$ in stop]))

data['nom'] = data['nom'].str.replace('[^| $\mid w \backslash s]$ ',', $)$

Pour dessiner le nuage des mots, utiliser par exemple les libraires et les instructions suivantes :

import matplotlib.pyplot as plt

from wordcloud import WordCloud, STOPWORDS

$\mathrm{t}=$ ",".join(str(i) if not isinstance( $\mathrm{i}$, unicode) else $\mathrm{i}$ for $\mathrm{i}$ in data["description"]) stop $=\operatorname{set}($ stopwords.words('french'))

wordcloud $=$ WordCloud(background_color="white", max_words=2000, stopwords=stop, contour_width=3, contour_color='steelblue').generate $(\mathrm{t})$

plt.imshow(wordcloud)

plt.axis('off')

plt.show()

\section{B. Traitement des données}

Une fois la base nettoyée, le texte converti en vecteurs, sont entamés le traitement et l'analyse des données. Trois étapes fondamentales sont suivies : l'analyse sentimentale permettant l'annotation de textes, la classification et enfin la prévision. Tous les codes détaillés ici pourraient être réécris avec les 
fonctions développées dernièrement sur Keras (Géron 2019). Voir par exemple le site RealPython ${ }^{5}$ pour des exemples de classification.

\section{a. Annotation}

Le code suivant produit, pour chaque description, un chiffre égal à 1 si la description est positive, -1 si elle est négative et 0 si elle est neutre.

from textblob import TextBlob

from textblob_fr import PatternTagger, PatternAnalyzer

def clean(text):

return, '.join(re.sub("(@[A-Za-Z0-9]+)|([^0-9A-Za-z It])|(|w+:WMS+)", " ", text).split())

def sentiment(text):

analysis $=\operatorname{TextBlob}($ clean $($ text $))$

if analysis.sentiment.polarity $>0$ :

return 1

elif analysis.sentiment.polarity $==0$ :

return 0

else:

return -1

Les valeurs calculées par sentiment sur data["description"] sont enregistrées dans un vecteur $\mathrm{y}$.

\section{b. Classification}

Les codes de classification de textes sont disponibles sur internet ${ }^{6}$. L'avantage de coder en Python est d'avoir une communauté très active et des codes en libre accès qu'il suffirait d'adapter aux données.

Ici, $X$ représente la colonne de description et y la classe associée $(0,1$ ou -1) selon que la description du produit est respectivement (neutre, positive, négative). Les données sont divisées en quatre parties : X_train, X_test, y_train et y_test. Le modèle est formé avec $80 \%$ des données (X_train, y_train). Il est testé avec les 20\% restants (X_test, y_test). Cette configuration fractionnée est pivotée quatre fois pour une couverture complète de l'ensemble de données.

from sklearn.model_selection import train_test_split

X_train, X_test, y_train, y_test $=$ train_test_split $(X, y$, test_size $=0.2$, random_state $=0$ )

Le code pour lancer l'algorithme de forêts aléatoires (Random Forest) est donné ci-dessous :

from sklearn.ensemble import RandomForestClassifier

\footnotetext{
${ }^{5}$ Exemples pour le traitement naturel du langage disponibles dans https://realpython.com/python-kerastext-classification/

${ }^{6}$ Voir par exemple https ://python.gotrained.com/tf-idf-twitter-sentiment-analysis/. La communauté scientifique et les développeurs sont très présents dans les forums de Stack Overflow ou github
} 
text_classifier $\quad=\quad$ RandomForestClassifier(n_estimators $=100$, random_state $=0$ )

text_classifier.fit(X_train, y_train)

Le code pour l'algorithme SVM (avec un noyau linéaire) est donné par :

from sklearn.svm import SVC

text_classifier $=$ SVC(kernel='linear' $)$

text_classifier.fit(X_train, y_train)

Ici, c'est l'algorithme SVM avec un noyau linéaire et utilisant la méthode de descente du gradient :

from sklearn.linear_model import SGDClassifier

text_classifier = SGDClassifier(loss='hinge', penalty='12', alpha=1e-3, $\mathrm{n} \_$iter $=5$, random_state $=42$ )

text_classifier.fit(X_train, y_train)

Le code pour l'apprentissage via les réseaux de neurones artificiels est donné ci-dessous :

from sklearn.neural_network import MLPClassifier

text_classifier $=$ MLPClassifier(activation='relu', alpha=1e-05, batch_size='auto', beta_1 $=0.9$, beta_2 $=0.999$, early_stopping $=$ False, epsilon=1e-08, hidden_layer_sizes $=(5$, 10), learning_rate $=$ 'constant', learning_rate_init $=0.001, \quad \max$ iter $=200$, momentum $=0.9, \quad$ n_iter_no_change $=10, \quad$ nesterovs_momentum $=$ True, power_t $=0.5$, random_state $=1$, shuffle $=$ True, solver $=$ 'lbfgs', tol $=0.0001$, validation_fraction $=0.1$, verbose $=$ False, warm_start $=$ False $)$

text_classifier.fit(X_train, y_train)

Le code pour l'algorithme Naive Bayes est :

from sklearn.naive_bayes import MultinomialNB

text_classifier $=$ MultinomialNB ()

text_classifier.fit(X_train, y_train)

c. Prévision

La commande text_classifier.predict permet de prédire la classe d'un nouveau texte.

predictions $=$ text_classifier.predict $($ X_test $)$

Les différentes mesures de performances (Exactitude, Précision, Rappel, $\mathrm{F}_{1}$ score, Matrice de confusion) sont données grâce au code suivant :

from sklearn.metrics import classification_report

from sklearn.metrics import confusion_matrix

from sklearn.metrics import accuracy_score

print(confusion_matrix(y_test,predictions))

print(classification_report(y_test,predictions))

print(accuracy_score(y_test, predictions)) 